\title{
Ultrasound-guided cannulation of hemodialysis access
}

\author{
Tadashi Kamata, Mayumi Tomita and Noriyuki lehara
}

\begin{abstract}
Because hemodialysis therapy cannot be performed without the cannulation of a vascular access, establishing a well-functioning vascular access is crucial. Recently, the number of patients with difficult arteriovenous (AV) fistula cannulation has increased due to changes in the epidemiology of the dialysis population. To address this issue, indications for real-time ultrasound-guided techniques have recently been reported. This review discusses methods of ultrasound-guided cannulation of dialysis access; it is difficult to cannulate a deep, small, or stiff vessel of an AV fistula on the first attempt with static ultrasound guidance in which the ultrasound probe remains stationary. Mastering a method in which the precise location of the needle tip can be continuously identified during the insertion by dynamic probe scanning is required. To achieve this, understanding the theory of ultrasound guidance, off/on-the-job training, and a sense of professionalism are important. The use of ultrasound also enables the safe catheter placement into a collapsed central vein and repeated direct puncture of a femoral vein. The latter reduces the risk of catheter-related blood stream infection by avoiding the use of an indwelling dual-lumen catheter. In conclusion, ultrasound-guided techniques enable precise vascular cannulation, which can result in significant patient and financial impact. The accumulation of further reports is required for the method to be acknowledged as the standard to cope with difficult vascular access for dialysis. As most of the latest ultrasound machines for procedural guidance are optimized for peripheral nerve block, a compact and affordable ultrasound device with image quality focused on vascular access is also needed.

Keywords: Arteriovenous fistula, Dynamic ultrasound guidance, Vascular access, Needle repositioning, Internal jugular vein, Guidewire, Femoral vein, Repeated cannulation, Double-lumen catheter, Point-of-care ultrasound
\end{abstract}

\section{Background}

The creation and maintenance of a well-functioning vascular access are crucial for efficient hemodialysis therapy. The native arteriovenous (AV) fistula is widely recognized as the vascular access of first choice for most hemodialysis patients in that it has a lower frequency of complications compared with other types of vascular access [1-3]. In addition to an older age, female gender, and a history of diabetes, obesity was shown to be a significant risk factor for failure to achieve an AV fistula [4]. As the KDOQI guideline states that a functional permanent access should be "less than $0.6 \mathrm{~cm}$ below the surface of the skin" [2], an AV fistula for obese patients may not be able to be cannulated with a traditional blind technique. On the other hand, the application of

\footnotetext{
* Correspondence: tkama@mtd.biglobe.ne.jp

Department of Nephrology, Kyoto City Hospital, 1-2, Higashi-takada-cho, Mibu, Nakagyo-ku, Kyoto 604-8845, Japan
}

ultrasound-guided vascular access, which is now the standard in central venous access, has expanded to peripheral vessels [5-7]. Similarly, in the field of dialysis, there are growing indications for its use in cases of difficult peripheral dialysis vascular access, although the number of reports is still limited [8-11]. Even in Japan, where the prevalence of obesity is lower than in other countries, difficult AV fistula access has increased because of the epidemic of diabetes and aging of the dialysis population. Multiple access attempts result in poor patient satisfaction and unnecessary costs [12] and so must be avoided. Thus, as ultrasound-guided puncture in difficult AV fistula access is described in the recent Japanese vascular access guideline [3], this method has attracted attention. Furthermore, as a study on daily hemodialysis suggested, frequent cannulation can influence the patency of an AV fistula [13]. An ultrasoundguided method may therefore have the potential to 
minimize AV fistula damage, resulting in a better prognosis for patients with difficult access. The aim of this review is to describe methods of ultrasound-guided cannulation of dialysis access and discuss conditions necessary for the methods to spread and help patients and dialysis providers avoid the problems of "poor access."

\section{Review}

\section{Peripheral venous access (AV Fistula)}

Unlike an AV graft, the accessibility of an AV fistula heavily depends on the condition of the patient's autogenous vessel, which often leads to difficult access with the traditional blind technique. Although buttonhole cannulation can be an option for difficult access [14], ultrasound-guided needle insertion can address this problem. Even if the AV fistula is difficult to cannulate on the initial cannulation, it may become possible to cannulate it with the traditional method by the improvement of edema or maturation of an AV fistula while continuing dialysis therapy with ultrasound guidance. The indications of ultrasound-guided AV fistula cannulation are as follows: a vessel which is too deep or small for reliable cannulation, a vessel with an adjacent artery or nerve, a vessel with a history of frequent multiple attempts, and a vessel whose cannulation on the first attempt is vital.

\section{Technique}

There are two approaches to visualize the needle: transverse and longitudinal imaging (Fig. 1). The majority of studies have used the transverse approach [9-11], and our preference is also this approach. Under transverse imaging, an operator can see the entire circumference of the vein and, thus, transverse imaging facilitates the cannulation of very small veins by enabling the operator to enter the vein right on the top of it. Additionally, there is a "one-operator technique" where one operator holds both a probe and needle and a "two-operator technique" with one holding the ultrasound probe and one performing the line placement procedure. The one-operator technique is advantageous in a busy clinical environment from the point of view of staff resources. Furthermore, with the one-operator technique, by scanning the probe dynamically with coordinated advancement of the needle, highly precise cannulation is possible under transverse imaging. The following is an example of dynamic ultrasound-guided peripheral vascular cannulation using transverse imaging and the oneoperator technique [9]. There are two points: firstly, the target vessel is thoroughly evaluated with ultrasound to determine the entry site. Ideally, there should be no artery or nerve adjacent to the entry site. Then, the entry site on the skin is determined based on the depth of the vessel and angle of the needle; as the distance from the probe edge and beam-generating site is $4-5 \mathrm{~mm}$ for most high-frequency linear probes, the distance should be considered in order to perform precise puncture (Fig. 2). Secondly, the angle between the probe and needle is controlled; the difficulty of puncturing a peripheral vein is in the fact that it lies in hyperechoic and heterogeneous subcutaneous tissue. The echo of the needle tip is visualized by diffraction of ultrasound, which scatters uniform waves with a relatively low amplitude in different directions, resulting in the difficulty of identifying the tip in a hyperechoic background. On the other hand, the shaft is visualized by reflection as the shaft is an
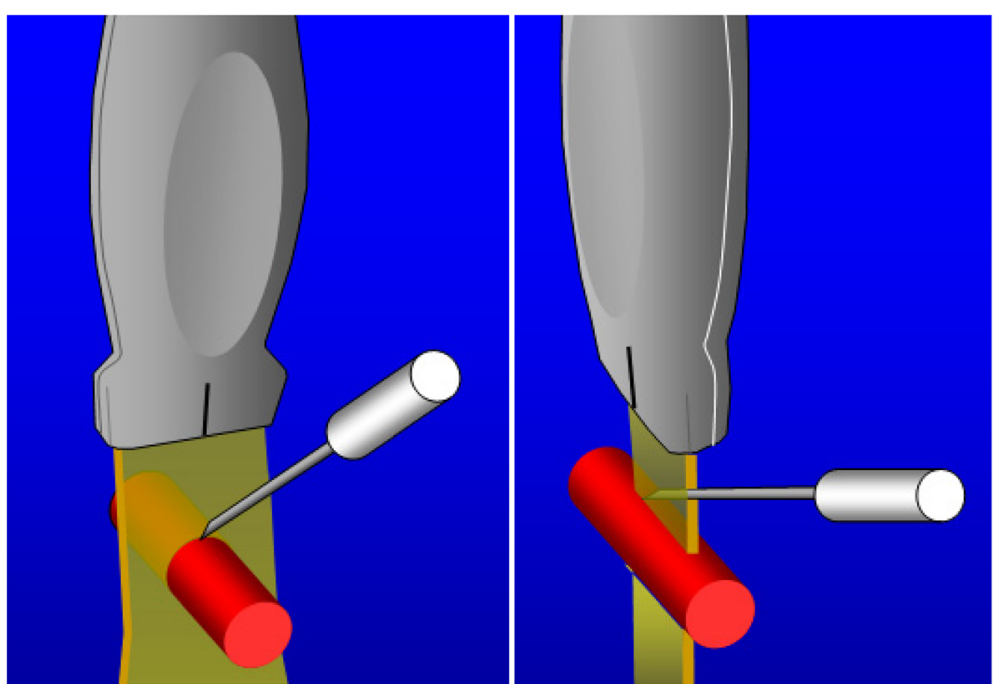

Fig. 1 Orientation of the probe in relation to the needle. Transverse approach (left) and longitudinal approach (right). Both methods have advantages and disadvantages 


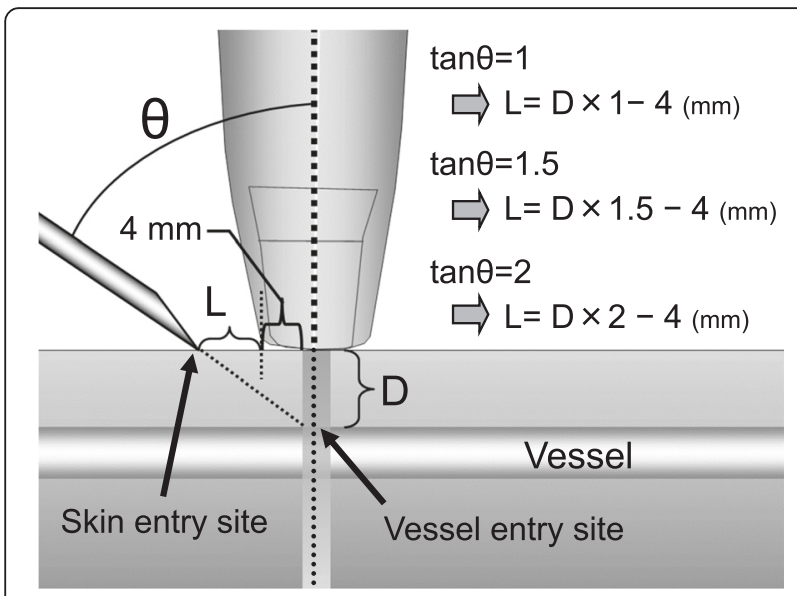

Fig. 2 Determination of the skin entry site. The skin entry site is easily determined from the depth of the vessel $(D)$ and angle of the needle $(\theta)$. Note the distance from the edge of the probe and the ultrasound-generating site. This distance may differ to some extent between probes. For example, if $\tan \theta$ is 1.5 and $D$ is $6 \mathrm{~mm}, L$ is calculated as $5 \mathrm{~mm}$. It is necessary to know the operator's own approximate insertion angle in advance

interface larger than the wavelength. By the rule of reflection, the shaft echo is maximized when ultrasound strikes the shaft at angles approaching $90^{\circ}$ [15], which is easy to observe on the ultrasound screen even in hyperechoic subcutaneous tissue. A detailed procedure using the theory described above is as follows (an additional movie file shows this in more detail (see Additional file 1)):

1. Observe the target vessel thoroughly with ultrasound. Veins can be easily differentiated from arteries based on compressibility, pulsatility, shape, and knowledge of anatomy.

2. Sterilize the skin entry site and probe, and apply a tourniquet on the arm. A sterilized probe cover and ultrasound gel should be used.

3. Administer local anesthesia if necessary.

4. Place the probe directly above the vessel entry site so that the center of the target vessel is visualized in the center of the ultrasound screen and logically determine the entry site of the skin.

5. Insert the 5-mm end of the needle at the center of the probe, and then, keeping the probe orthogonal to the needle, move the probe backward to visualize the shaft image. Advance the probe forward and stop as soon as the bright spot has disappeared (Fig. 3). This point can be judged as the location of the needle tip. Subsequently, advance the needle and then identify the new location of the tip by moving the probe to-and-fro down the course of the vessel. If the trajectory of the shaft image misses the center of the target vessel, redirect the needle by angling it sideways. Repeat those steps until the needle tip is

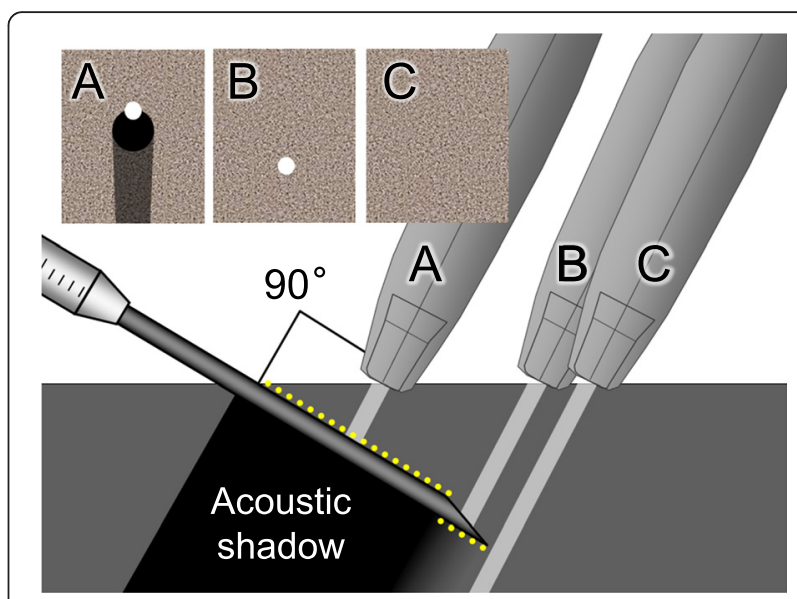

Fig. 3 Identification of the needle tip by dynamic scanning. By adjusting the probe perpendicular to the needle shaft, the echogenicity of the shaft (dotted line) markedly increases. With the forward movement of the probe, the ultrasound image changes as indicated (inset, $A$ to $C$ ). Strictly speaking, the precise location of the needle tip is between $B$ and $C$

directly on the top of the vessel. Tenting of the anterior wall of the vessel is also a sign that the tip has reached the anterior wall.

6. Enter the vessel and visualize the bright spot within its lumen by advancing the probe carefully.

(Although blood return may be noted at this time, it is ancillary information under ultrasound guidance as long as favorable imaging is being maintained.) While lowering the angle of the needle, advance the probe until the bright spot disappears within the vessel, and then advance the needle until the bright spot appears within it (an additional movie file shows this in more detail (see Additional file 2)). By repeating the above-described maneuver, guide the needle tip within the lumen of the vessel without touching the vessel's inner wall. When using the catheter-over-needle method, advance the needle to the point where the tip of the catheter is well within the lumen of the vessel. Then, put the probe down and advance the catheter fully over the needle. Before advancing the catheter, the operator may ensure successful cannulation with a longitudinal image if needed [10]. When a needle without a catheter is used, the tip should be guided to a final position; otherwise, it may go through the posterior or side wall of the vessel even after blood return is observed.

Longitudinal imaging is frequently used in ultrasoundguided peripheral nerve block [16]. Under longitudinal imaging, an operator can see the entire length of the needle as it advances. However, as it is sometimes 
difficult to keep the center of the vessel perfectly aligned with the needle shaft within the thin ultrasound beam, small vessels can be very challenging using this imaging. Taken together, although still controversial from an evidence-based point of view, we support the use of transverse imaging as the main method and longitudinal imaging as an auxiliary method. However, even if largescale randomized control studies facilitate a general consensus in the future, it may be fair to say that it is ultimately a personal choice because clinical settings and individual traits are quite diverse in reality. If a person feels satisfied with one method in particular, that is the main method to use.

\section{Ultrasound-guided needle repositioning}

In spite of its marked precision, it is not practical to apply ultrasound guidance to all AV fistulae because of a limited number of ultrasound machines, time, and labor. When placing a needle with the traditional method and the operator judges that the insertion is probably going to fail (i.e., no blood return, resistance to catheter advancement, etc.), then ultrasound guidance can be started to correct the needle tip location. Importantly, this not only prevents the failure of needle placement but also tells the operator why he or she almost failed [17]. Thus, an ultrasound machine can be a good training tool for the traditional puncture method. Although there may be fear that introducing ultrasound machine use could erode traditional puncture skills, it depends on the attitude of the dialysis providers. Dialysis providers should continue to train themselves in both techniques. Of note, when ultrasound-guided needle repositioning is used, some kind of physical barrier, such as a dedicated cover, surgical gloves, film dressing, and commercially available plastic wrap [18], must be applied to the probe head to prevent potential cross-contamination between patients. However, as an experienced operator can perform the procedure without exposing the skin entry site to ultrasound gel, sterilized ultrasound gel is not always necessary.

\section{Central venous access}

Ultrasound is also useful in central venous cannulation in the field of hemodialysis, as discussed below.

\section{Internal jugular vein}

The effectiveness of an ultrasound-guided internal jugular venous approach to facilitate central venous access and improve patient safety has been shown in a metaanalysis [19]. This is at least in part due to the fact that the internal jugular vein is relatively near the surface of the skin, and the hypoechoic sternocleidomastoid muscle lies anterior to it. Basically, the ultrasoundguided technique for the internal jugular vein is similar to that for a peripheral vein except for three points: firstly, the angle between the probe and needle shaft should not necessarily be orthogonal because the needle tip can be relatively easily identified as the needle traverses the hypoechoic sternocleidomastoid muscle. Instead, jiggling (subtle back-and-forth movements) of the needle is effective as moving objects are easier to recognize than stationary ones. Secondly, successful placement of the needle inside the jugular vein should be ensured by adding the use of longitudinal imaging routinely for safety (Fig. 4). It is common knowledge for a sonographer to observe an object from different directions. Thirdly, due to marked puncture resistance [20] or hypovolemia, access may be difficult even if the Valsalva maneuver is applied. In such cases, the "Push and stick" technique, which enables penetration of only the anterior wall by pressing the anterior wall with the needle tip and subsequent quick forward movement of the needle, is effective [21] (an additional movie file shows this in more detail (see Additional file 3)). If one is solely dependent on blood return to judge the entry of the needle tip into the lumen of the vessel, a collapsed central vein can be very challenging.

Although the right internal jugular vein is recommended as an approach of first choice in the guidelines $[2,3]$, this approach can cause life-threatening

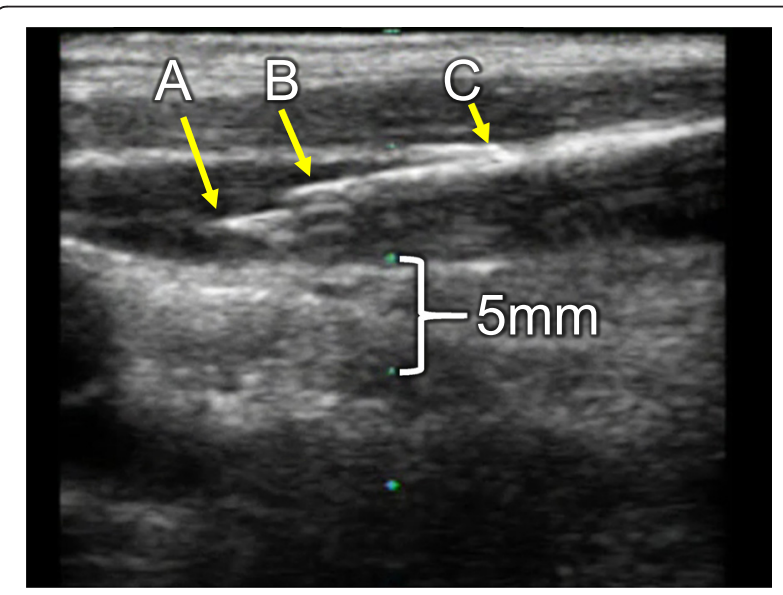

Fig. 4 Longitudinal image of a needle within the internal jugular vein. After advancing a needle for the catheter-over-needle technique into a rather collapsed jugular vein under transverse imaging, the probe is rotated $90^{\circ}$ to obtain a longitudinal image. This not only ascertains the intravenous placement of the needle tip (A) but also ensures the intravenous placement of the catheter tip (B). By measuring the distance between the tips of the needle and catheter in advance, the precise location of the catheter tip can be determined on the ultrasound screen. $C$ is the entry site of the vein. Just before the removal of the needle and insertion of the guidewire, the distance from $B$ to $C$ should be at least $5 \mathrm{~mm}$ to facilitate guidewire insertion. However, one must not advance the needle too deeply because the deeper the needle advances, the more obscure the image becomes in most cases of central veins 
complications due to inadvertent arterial puncture as the internal jugular vein is located adjacent to vital structures. A needle might go through the internal jugular vein and hit arteries, albeit with the use of ultrasound guidance [22]. The diameters of a central venous duallumen catheter and its dilator are larger than those of the introducer needle. It is, therefore, crucial to rule out inadvertent arterial guidewire misplacement before inserting the dilator [23], especially in the case of hemodialysis dual-lumen catheters whose diameter is much larger than that of the other types of central venous catheter. Although checking blood return is informative, it is not always reliable due to a patient's condition. To cope with this, after inserting a guidewire into the internal jugular vein, ensuring successful placement of the guidewire inside the lumen of the target vein with ultrasound is feasible and effective: in a recent study in which a transverse ultrasound view of the guidewire was traced proximal to the heart by tilting an ultrasound probe at the supraclavicular fossa, the authors noted successful intravenous placement of the guidewire in all 10 cases tested [24]. To effectively utilize this technique, the use of a large spring guidewire (diameter $\geq 0.89 \mathrm{~mm}$ ) which generates strong diffraction of ultrasound waves is necessary (Fig. 5). Conveniently, large guidewires are considered to cause less kinking on inserting a dilator, and using a catheter-over-needle system minimizes the risk of damaging the spring wire. The concept of "The smaller the needle and guidewire, the safer", which emerged before the era of ultrasound guidance, should not be taken for granted, especially in the case of large-sized multiple-lumen catheters.

\section{Femoral vein}

Because of its anatomical location, femoral venous access carries fewer risks of fatal mechanical complications than other types of central access. The femoral vein is preferred as an insertion site in emergency settings like orthopnea and shock, since this approach can be achieved comparatively easier and faster in critically ill bedridden patients. Disadvantages are high risks of infection and arterial puncture [25]. Although there is a report that an ultrasound-guided technique significantly improved the success rate, reduced the number of attempts, and decreased the incidence of complications related to femoral venous dialysis catheter insertion [26], the first-attempt success rate under ultrasound guidance in this study was still $85 \%$. This is at least in part because femoral veins are located under a hyperechoic heterogeneous subcutaneous tissue, resulting in difficulty visualizing the needle tip directly on the ultrasound screen, like peripheral veins. The problem is that if one tries to increase the echogenicity of the needle shaft by adjusting the probe perpendicular to the needle, the
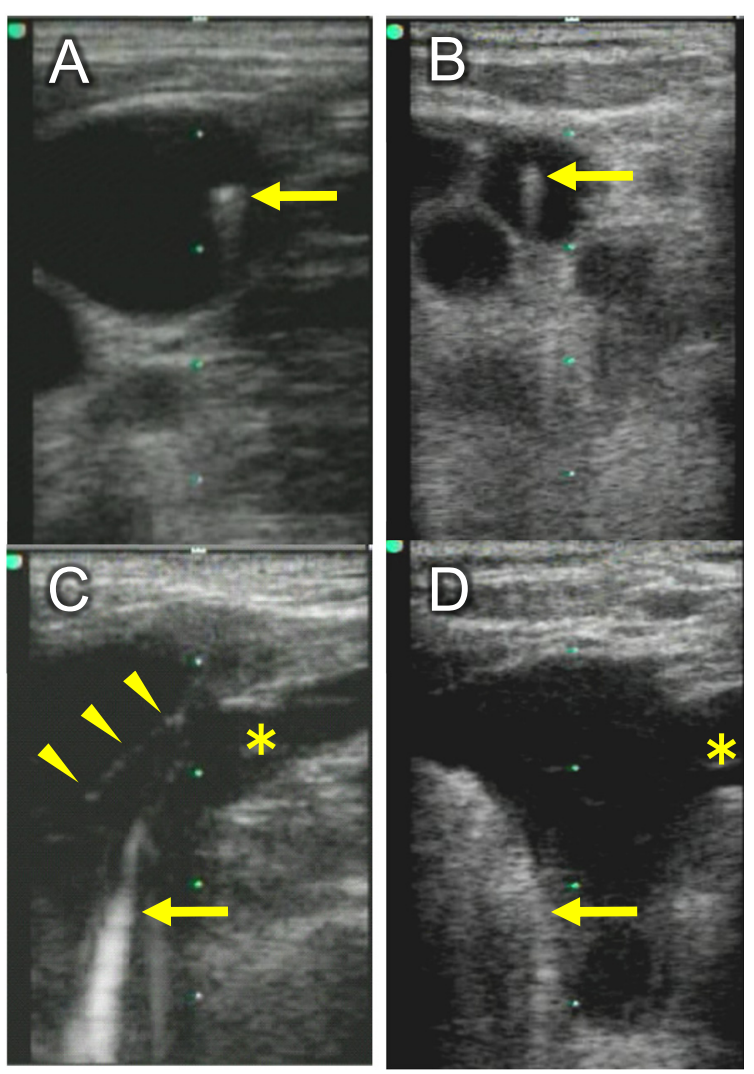

Fig. 5 Ultrasound images of $\varphi 0.97-\mathrm{mm}(\mathbf{a}, \mathbf{c})$ and $\varphi 0.64-\mathrm{mm}(\mathbf{b}, \mathbf{d})$ guidewires. $\mathbf{a}, \mathbf{b}$ : Images near the puncture site with the probe kept almost upright. Guidewires can be seen in the internal jugular vein. c, d: Images at the supraclavicular fossa with the probe tilted almost horizontally. The guidewires are visible within the brachiocephalic vein, which warrants the subsequent insertion of a dilator. Without tilting the probe under transverse imaging, the guidewire in the deep portion cannot be visualized due to interference from the clavicle. Note that the visibility of the large spring wire is more favorable. Arrow: guidewire; asterisk: subclavian vein; arrowheads: venous valve between the internal jugular and brachiocephalic veins

image of the femoral vein becomes obscure as the angle of the needle is steep in the case of deep veins.

There are two points to facilitate the cannulation of deep veins (depth $>1 \mathrm{~cm}$ ) which are surrounded by hyperechoic tissue: firstly, the shaft of the needle is relatively easy to recognize because the acoustic shadow of the shaft is conspicuous in the hyperechoic subcutaneous tissue. Fortunately, the acoustic shadow is clear, especially with a large needle like hemodialysis needles. Being similar to the method described for the deep peripheral vein, a shaft image can be traced by to-and-fro scanning. The approximate location of the tip can be judged as the point where the shaft echo vanishes during forward scanning. Additionally, if the trajectory of the acoustic shadow is always directed to the center of the femoral vein, the tip of the needle will reach there 
without fail (Fig. 6) (an additional movie file shows this in more detail (see Additional file 4)). Secondly, even if the needle tip is not directly visible, its location can be identified by needle jiggling, which causes the movement of the surrounding soft tissue. When the ultrasound beam meets the tip, the movement of the surrounding soft tissue becomes the most rigorous. Furthermore, if the tip reaches the anterior wall, tenting of the vein appears, which is also a helpful sign. Utilizing those methods, 16 patients underwent blood purification by repeated ultrasound-guided femoral vein puncture until either peripheral vascular access was established or blood purification was withdrawn [27]. In this study, a 16-gauge, 7.5-cm-long dialysis catheter was inserted into the femoral vein at every blood purification session as the blood-drawing site under ultrasound guidance with the catheterover-needle technique (Fig. 7). As a rule, peripheral veins were used as blood-returning sites. The authors reported that 156 blood purifications (1-48/person, 9.8 sessions on average) were performed effectively using this method without new-onset blood stream infection or hematoma formation. Thus, ultrasoundguided repeated femoral vein puncture can be a useful option as a temporary access. It prevents the inadvertent puncture of impalpable arterial branches which can be a cause of hematoma formation. Moreover, the use of a problematic indwelling dual-lumen catheter can be avoided. A caveat specific to this method is that operators must ensure successful puncture with the utmost caution if the femoral vein is used as a blood-returning site. Needle tip displacement outside of the vein will cause marked retroperitoneal hematoma.

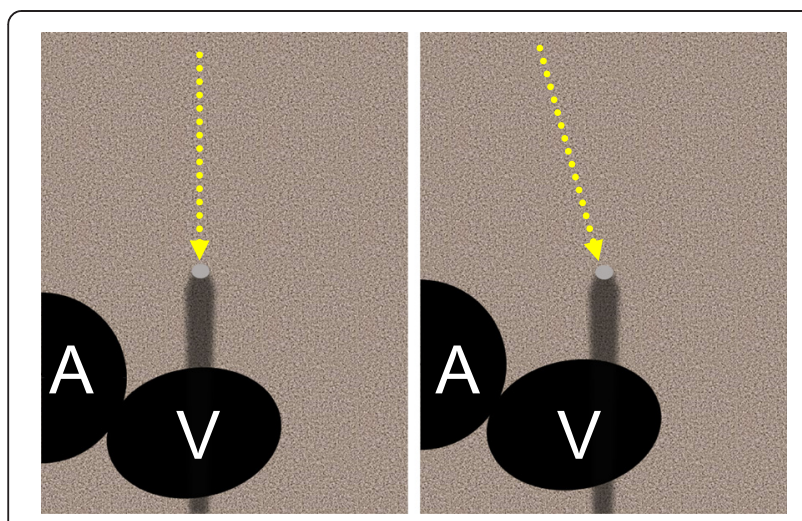

Fig. 6 Acoustic shadow-tracing method. If the probe is scanned to-and-fro down the course of the femoral vein, and the trajectory of the shaft image (dotted arrow) is directed to the center of the femoral vein, the direction of the needle is correct (left). Otherwise (right), redirection of the needle is necessary. A: femoral artery $V$ : femoral vein

\section{Training method}

Simply using an ultrasound-guided technique does not necessarily mean safety [28]. Naturally, training is required. There are three prerequisites for a training program: firstly, the theory of ultrasound-guided cannulation must be understood. Otherwise, the procedure would be intuitive, which cannot be a reliable method. In addition, a logical method is easier to learn than an intuitive one. Secondly, not only on-the-job training with an instructor but also off-the-job training with a simulator is essential. A recent meta-analysis showed that in comparison with no intervention, simulation training in health professionals' education is associated with marked effects on knowledge, skills, and behaviors and moderate effects on patient-related outcomes [29]. Thirdly, reflection after on-the-job training is necessary. Novice dialysis providers can educate themselves by looking back upon both good and bad aspects of their procedure. The reflection step can be effectively facilitated by watching the video of the ultrasound screen recorded during onthe-job training [30]. Recently, a compact video recorder with the size of a matchbox is commercially available.

An expensive high-fidelity simulator is not necessarily more effective than a cheap low-fidelity one, especially for novice trainees [31]. As commercially available simulators are expensive and non-durable, the use of a handmade simulator is recommended (Fig. 8). The method to construct the simulator is as follows [32]:

1. To make mock vessels, build tunnels through a hard, cuboid konjac jelly (traditional Japanese food available in grocery stores) by pressing a large straw into it.

2. Place the jelly in a tray whose depth is approximately the same as the thickness of the jelly.

3. Pour water into the tray until the top of the jelly is submerged.

4. Remove the air within the tunnels by squeezing the superior surface of the jelly with fingers.

\section{Ultrasound equipment}

Electromagnetic needle tracking systems which indicate the place where the needle tip will appear on an ultrasound screen under transverse imaging are available [33]. However, these will not widely spread in dialysis therapy for the time being because of cost, precision, labor demands and the size of the equipment. Furthermore, even if under the use of those systems, expertise in sonoanatomy and reasonable needle handling skills are still mandatory, especially in the case of a small or collapsed vein. An ultrasound machine that is applicable to both central and peripheral veins in a dialysis room is considered to fall into the category of point-of-care ultrasound [34]. Prerequisites for an ideal vascular access ultrasound machine are discussed below. 


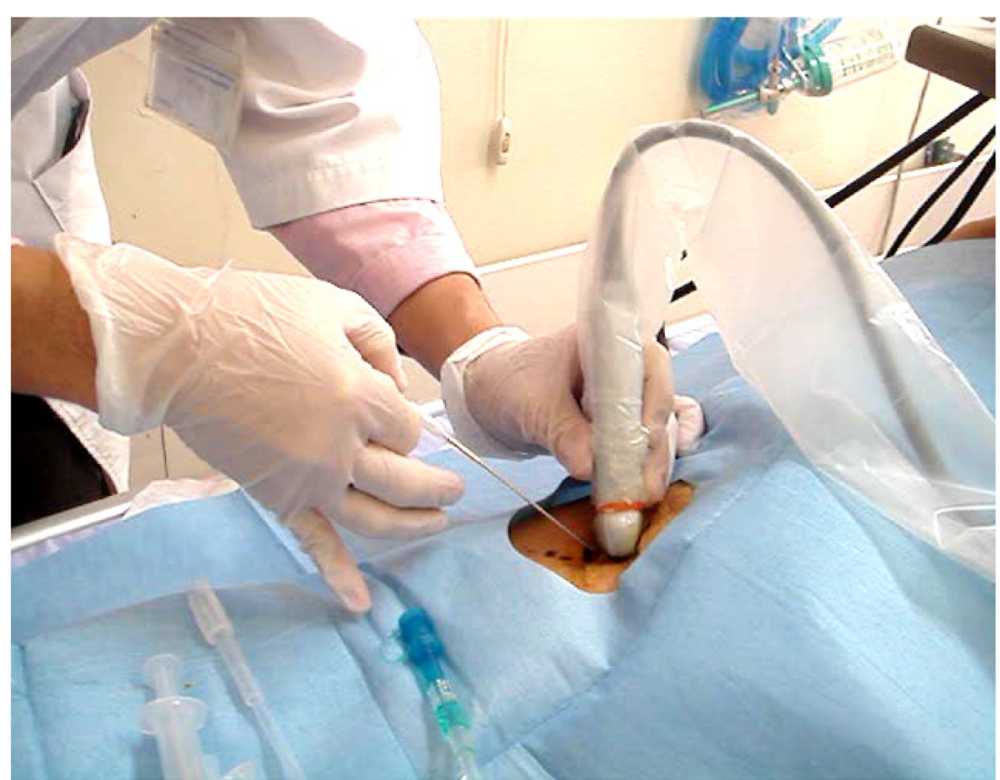

Fig. 7 Ultrasound-guided femoral vein puncture. Note that the angle of the needle is steeper than that of the peripheral vein puncture. The probe is perpendicular to the skin, but not to the needle

\section{Size and durability}

In some countries like Japan, most patients receive hemodialysis in bed. When a conventional consolestyle ultrasound machine is used, the installation site of the machine is limited because of the machine's size and limited working space. In this situation, operators have to change their gaze during needle insertion, which makes the procedure ergonomically stressful. Although some facilities have utilized a head-mounted display [35] to overcome this issue, the size of an ultrasound machine should be compact so that it can be placed next to the patient on the bed. For patients receiving dialysis in a chair, a rollaway stand that can be easily attached and detached should also be available.

As ultrasound machines get smaller, there is a greater risk of them falling in busy dialysis facilities. Therefore, toughness is necessary.

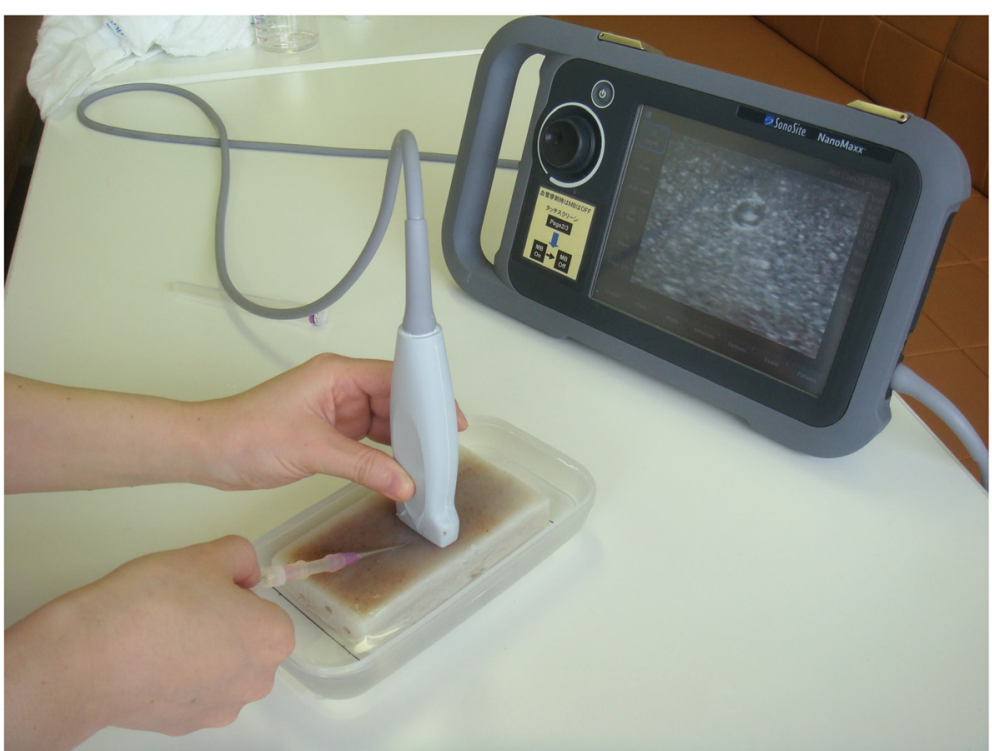

Fig. 8 Handmade simulator for ultrasound-guided vascular access. By utilizing traditional Asian food, a cheap and durable simulator can be instantly made 


\section{Image quality}

For the safety reasons mentioned above, image quality that delineates a clear acoustic shadow is desirable so that a shaft is not mistaken for a tip under transverse imaging. In addition, high time-resolution is a prerequisite because needle jiggling, which is an essential technique in central venous access, does not work under low time-resolution imaging. Many of the latest ultrasound machines are installed with spatial-compound imaging where a couple of consecutive still image frames with different incident angles are averaged to improve spatial resolution [36]. With this function turned on, timeresolution becomes impaired and the acoustic shadow is obscured. Therefore, it is better to turn the spatialcompound imaging off in vascular access.

\section{Affordability}

Because most vascular access cannulations are done in a short period of time in typical hemodialysis facilities, introducing more than one ultrasound machine into a dialysis room would facilitate the smooth performance of daily routine work. Consequently, vascular access machines should be affordable.

\section{Educational function}

Recently, some ultrasound machines have been installed with instruction images and videos of ultrasound-guided procedures. This is, however, not necessarily effective, because informative text books and instruction videos are readily available. Video output sockets (both digital and analog) are needed to record the ultrasound screen on video from an educational point of view, as stated in the "Training method" section.

\section{Conclusions}

First and foremost, creating a well-functioning vascular access and its maintenance are of the utmost importance. Needless to say, the traditional blind cannulation of a peripheral vascular access is a fundamental technique. Nevertheless, ultrasound-guided vascular access can be an effective method in continuing adequate hemodialysis therapy for some patients. The accumulation of further reports is required for the method to be acknowledged as the standard to cope with difficult AV fistula access.

Ultrasound-guided vascular access has three pillars: theory, off/on-the-job training, and an ultrasound machine optimized for vascular access. Among these, the former two require a strong sense of professionalism so that inserters achieve vascular access on the first attempt by any means. Finally, ultrasound machine manufacturers should realize that nerve block, which is performed exclusively under longitudinal imaging, and vascular access under transverse imaging are quite different in terms of their methodology. Therefore, a compact and affordable ultrasound machine with the image quality focused on vascular access is needed.

\section{Additional files}

\begin{abstract}
Additional file 1: Ultrasound-guided deep peripheral vein cannulation. The brachial vein (depth $=5 \mathrm{~mm}$ ) of the mid-arm is cannulated as the blood-returning site under real-time ultrasound guidance. Normally, this vein is not punctured because it is usually invisible, impalpable, and sandwiched between the median nerve and brachial artery. Without dynamic probe scanning, precise and safe cannulation of this vein is difficult. (MP4 13095 kb)
\end{abstract}

Additional file 2: Control of a needle tip within the lumen of a vessel. With this maneuver, the needle tip can be advanced inside the vessel sufficiently without damaging its inner wall to ensure line placement for dialysis. This maneuver is especially effective for vessels with a small diameter, an easy collapsibility, or a high puncture resistance. (MP4 $7811 \mathrm{~kb}$ )

Additional file 3: Needle jiggling and "Push and stick" technique. A collapsed internal jugular vein is targeted. The Valsalva maneuver is impossible due to the patient's condition. While jiggling, the needle is redirected to the left. After the needle tip has reached the center of the anterior wall of the vein, the "Push and stick" technique is performed. Immediately after the "Push and stick" maneuver, the needle tip is actually in but just barely. At this point and time, the operator can start another maneuver, which was described for peripheral vessels, to safely advance the needle tip several millimeters further within the lumen of the internal jugular vein. These manipulations enable placement of the catheter tip of the introducing needle within the vein without fail, which ensures subsequent guidewire advancement. (MP4 2772 kb)

Additional file 4: Ultrasound-guided femoral vein cannulation. A 3.5-cm-deep femoral vein is cannulated with a long 16-gauge dialysis needle by the catheter-over-needle technique. The needle tip is guided precisely to on the top of the vein without hitting the adjacent artery which lies to its upper left. After entering the femoral vein, the needle tip is again guided inside the lumen of the vein to ensure that the catheter tip is well within the lumen of the vessel, which facilitates subsequent catheter advancement. (MP4 12434 kb)

Abbreviation

AV: arteriovenous.

Competing interests

The authors declare that they have no competing interests.

Authors' contributions

TK prepared the manuscript. MT and NI helped to draft the manuscript. All authors read and approved the final manuscript.

Received: 28 August 2015 Accepted: 4 November 2015

Published online: 19 February 2016

\section{References}

1. Feldman $\mathrm{HI}$, Kobrin S, Wasserstein A. Hemodialysis vascular access morbidity. J Am Soc Nephrol. 1996;7:523-35.

2. National Kidney Foundation. KDOQI 2006 vascular access guidelines. 2006. https://www.kidney.org/sites/default/files/docs/12-50-0210_jag_dcp_ guidelines-va_oct06_sectionc_ofc.pdf. Accessed 2 Oct 2015.

3. Kukita K, Ohira S, Amano I, Naito H, Azuma N, Ikeda K, et al. 2011 update japanese society for dialysis therapy guidelines of vascular access construction and repair for chronic hemodialysis. J Jpn Soc Dial Ther. 2011;44:855-938 (in Japanese).

4. Ethier J, Mendelssohn DC, Elder SJ, Hasegawa T, Akizawa T, Akiba T, et al. Vascular access use and outcomes: an international perspective from the dialysis outcomes and practice patterns study. Nephrol Dial Transplant. 2008;23:3219-26. 
5. Egan G, Healy D, O'Neill H, Clarke-Moloney M, Grace PA, Walsh SR Ultrasound guidance for difficult peripheral venous access: systematic review and meta-analysis. Emerg Med J. 2013;30:521-6.

6. Roberts J, Manur R. Ultrasound-guided radial artery access by a nonultrasound trained interventional cardiologist improved first-attempt success rates and shortened time for successful radial artery cannulation. J Invasive Cardiol. 2013;25:676-9.

7. Pittiruti M, Scoppettuolo G, Emoli A. Parenteral nutrition through ultrasound-placed piccs and midline catheters is associated with a low rate of complications: an observational study. Nutr Ther Metabol. 2009;27:142-8.

8. Hanafusa N, Kondo Y, Kaneko T, Niwa T, Yamamoto H, Watanabe Y, et al. Vascular access puncture method with guidance by a portable ultrasonographic device. J Jpn Soc Dial Ther. 2007;40:517-21 (in Japanese)

9. Kamata T, Ochiai M, Osaki K, Fujisawa N, Kadoya Y, Yashiro M. Ultrasoundguided brachial venous cannulation as a novel venous needle site in hemodialysis patients. J Jpn Soc Dial Ther. 2011;44:237-43 (in Japanese).

10. Hanafusa N, Noiri E, Nangaku M. Vascular access puncture under ultrasound guidance. Ther Apher Dial. 2014;18:213-4.

11. Patel RA, Stern AS, Brown M, Bhatti S. Bedside ultrasonography for arteriovenous fistula cannulation. Semin Dial. 2015;28:433-4

12. Lee T, Barker J, Allon M. Needle infiltration of arteriovenous fistulae in hemodialysis: risk factors and consequences. Am J Kidney Dis. 2006:47:1020-6.

13. Suri RS, Larive B, Sherer S, Eggers P, Gassman J, James SH, et al. Risk of vascular access complications with frequent hemodialysis. J Am Soc Nephrol. 2013;24:498-505.

14. Wong B, Muneer M, Wiebe N, Storie D, Shurraw S, Pannu N, et al. Buttonhole versus rope-ladder cannulation of arteriovenous fistulas for hemodialysis: a systematic review. Am J Kidney Dis. 2014;64:918-36.

15. Myers K, Clough A. Making sense of vascular ultrasound. London: Arnold; 2004. p. 3-4.

16. Nagdev A, LeVine S, Mantuani D. Point-of-care ultrasound. Philadelphia: Elsevier; 2015. p. 271-82.

17. Kamata T, Ochiai M, Fujisawa N, Kadoya Y, Tomita M. Hemodialysis and portable ultrasound. J Kyoto City Hosp. 2012;32:1-7 (in Japanese).

18. Garland SM, Newnan DM, de Crespigny LC. Plastic wrap for ultrasound transducers. Herpes simplex virus transmission. J Ultrasound Med. 1989;8:661-3.

19. Hind D, Calvert N, McWilliams R, Davidson A, Paisley S, Beverley C, et al. Ultrasonic locating devices for central venous cannulation: meta-analysis. BMJ. 2003;327:361

20. Adachi YU, Tuzuki M, Matsuda N. Is it constantly possible to penetrate only the anterior vessel wall against hydrostatic strain? Crit Care Med. 2012:40:2534-5.

21. Tokumine J. Manual of central venous catheterization using ultrasound guidance. Tokyo: Sogo Igaku sha; 2007. p. 26-7 (in Japanese).

22. Blaivas M, Adhikari S. An unseen danger: frequency of posterior vessel wall penetration by needles during attempts to place internal jugular vein central catheters using ultrasound guidance. Crit Care Med. 2009;37:2345-9.

23. Stone MB, Nagdev A, Murphy MC, Sisson CA. Ultrasound detection of guidewire position during central venous catheterization. Am J Emerg Med. 2010;28:82-4.

24. Kamata T, Ochiai M, Fujisawa N, Kadoya Y. Visibility of guidewires in ultrasound-guided internal jugular double lumen catheterization. J Jpn Soc Dial Ther. 2012:45:475-82 (in Japanese).

25. McGee DC, Gould MK. Preventing complications of central venous catheterization. N Engl J Med. 2003;348:1123-33.

26. Prabhu MV, Juneja D, Gopal PB, Sathyanarayanan M, Subhramanyam S, Gandhe S, et al. Ultrasound-guided femoral dialysis access placement: a single-center randomized trial. Clin J Am Soc Nephrol. 2010;5:235-9.

27. Kamata T, Shu S, Ochiai M, Osaki K, Fujisawa N, Kadoya Y, et al. Blood purification therapy using repeated ultrasound-guided femoral vein puncture: report of sixteen cases. J Jpn Soc Dial Ther. 2012;45:241-6 (in Japanese).

28. Blaivas M. Video analysis of accidental arterial cannulation with dynamic ultrasound guidance for central venous access. J Ultrasound Med. 2009;28:1239-44.

29. Cook DA, Hatala R, Brydges R, Zendejas B, Szostek JH, Wang AT, et al. Technology-enhanced simulation for health professions education: a systematic review and meta-analysis. JAMA. 2011;306:978-88.
30. Kamata T, Ochiai M, Fujisawa N, Kadoya Y, Tomita M, Okamura M. Simulation program for ultrasound-guided central venous access procedura training. J Jpn Soc Dial Ther. 2012;45:1027-33 (in Japanese).

31. Alessi S. Fidelity in the design of instructional simulations. Journal of Computor-Based Instruction. 1988;15:40-7.

32. Kamata T. One-coin simulator for ultrasound-guided vascular access. The Japanese Journal of Dialysis and caring. 2010;19:6-7 (in Japanese).

33. Choquet $\mathrm{O}$, Abbal B, Capdevila X. The new technological trends in ultrasound-guided regional anesthesia. Curr Opin Anaesthesiol. 2013;26:605-12.

34. Moore $\mathrm{CL}$, Copel JA. Point-of-care ultrasonography. N Engl J Med. 2011;364:749-57.

35. Udani AD, Harrison TK, Howard SK, Kim TE, Brock-Utne JG, Gaba DM, et al. Preliminary study of ergonomic behavior during simulated ultrasound-guided regional anesthesia using a head-mounted display. J Ultrasound Med. 2012;31:1277-80.

36. Szabo T. Diagnostic ultasound imaging: inside out. Burlington: Elsevier; 2004. p. 327-9.

\section{Submit your next manuscript to BioMed Central and take full advantage of:}

- Convenient online submission

- Thorough peer review

- No space constraints or color figure charges

- Immediate publication on acceptance

- Inclusion in PubMed, CAS, Scopus and Google Scholar

- Research which is freely available for redistribution

Submit your manuscript at www.biomedcentral.com/submit 